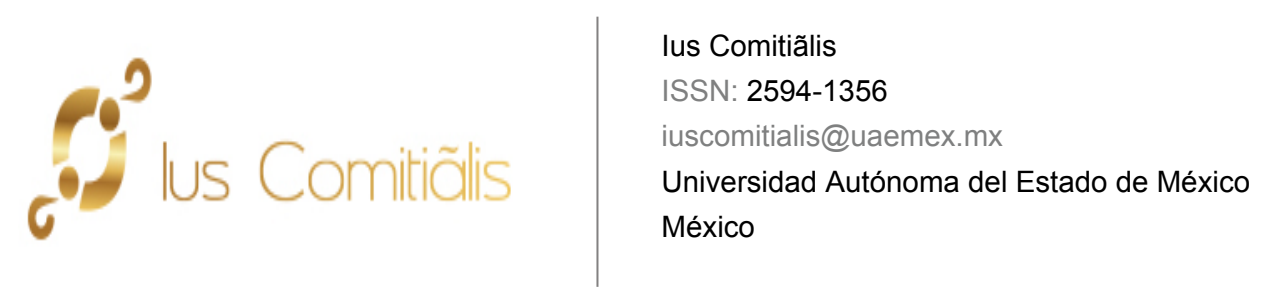

Ríos Ruíz, Alma de los Ángeles

Consideraciones en torno al Plan Nacional de Desarrollo 2019-2024

Ius Comitiãlis, vol. 2, núm. 4, 2019, Julio-Diciembre, pp. 29-46

Universidad Autónoma del Estado de México

México

DOI: https://doi.org/10.36677/iuscomitialis.v2i4.12944

\title{
Número completo
}

Más información del artículo

Página de la revista en redalyc.org 


\section{Consideraciones en torno al Plan Nacional de Desa- rrollo 2019-2024 \\ Considerations about the National Development Plan 2019-2024}

Alma de los Ángeles Ríos Ruiz*

Resumen El presente trabajo tiene como objetivo analizar las políticas públicas en el Estado Mexicano a la luz del Plan Nacional de Desarrollo 2019-2024, por ser el documento que refleja la situación actual de nuestro país e identifica los principales problemas y demandas de la sociedad, de ahí se desprende que su estudio busque comprender, tanto los principios y ejes como los objetivos que en él se concentran, para conocer, comprender las acciones y rutas de desarrollo que serán implementadas durante el actual sexenio a través de políticas públicas o acciones de gobierno tendientes a resolver los problemas nacionales que frenan el desarrollo del país con la finalidad de abatir los índices de pobreza, resolver pacíficamente conflictos, fortalecer las fuerzas armadas en materia de seguridad, consolidad la cooperación con los distintos países de las regiones del mundo, contribuir al desarrollo humano sostenible de México, entre otros aspectos.

Palabras clave Plan Nacional de Desarrollo, Desarrollo Sostenible, Proyecto de Nación, Políticas Públicas, Programas Sociales.

Abstract The present work aims to analyze public policies in the Mexican state in light of the National Development Plan 2019-2024 for being the document which reflects the current situation of our country and identifies the main problems and demands of society, Its study seeks to understand both the principles, axes, as well as objectives that are within it to know, understand the actions and development routes that will be implemented during the current sexennium through public policies or government actions aimed to solving national problems that slow down the development of the country in order to reduce poverty rates, resolve conflicts peacefully, strengthen the armed forces in terms of security, consolidate cooperation with the different countries of the regions of the world, contribute to the sustainable human development of Mexico, among other aspects.

Key words National Development Plan, Sustainable Development, Nation Project, Public Policies, Social Programs.

*Doctora en Derecho. Profesora de tiempo completo "C", Universidad Nacional Autónoma de México, México. Miembro del Sistema Nacional de Investigadores (SNI) nivel 1. ORCID https://orcid.org/0000-0003-3939-0600. Correo electrónico: alma_rios_r@hotmail.com 


\section{INTRODUCCIÓN}

Las políticas públicas vistas como una forma particular de decidir y ejecutar las acciones de gobierno varían de un país a otro dependiendo del sistema político de cada nación, de la tradición jurídica, cultura local, sobre todo de los problemas imperantes que requieren atención para permitir el desarrollo de cada nación; en el caso que nos ocupa del Estado Mexicano, el gobierno de nuestro país planifica sus políticas con base en el Plan Nacional de Desarrollo (PND).

El PND es el documento en donde el Gobierno de México, a través de consultar a la población, explica cuáles son sus objetivos prioritarios durante el sexenio, en este caso para el periodo 2019-2024, su meta es establecer y orientar el trabajo que deberán realizar los servidores públicos los próximos seis años, para lograr el desarrollo del país, el bienestar de los mexicanos y hacer de México un país más próspero, justo e incluyente para todos los ciudadanos (Centro Nacional de Control de Energía, 2019).

La participación social en el marco de la creación del PND tiene por objeto informar y enriquecer la construcción de las políticas públicas para que éstas se encuentren alineadas a los intereses y necesidades de los distintos actores sociales, por ello se permite que las opiniones de las entidades federativas, municipios, grupos, comunidades indígenas, órganos autónomos, grupos prioritarios, académicos, empresarios, ciudadanos, dependencias APF y el Congreso, estén reflejadas en elaboración, actualización, ejecución del plan, así como en los programas derivados del mismo.

La estructura del PND 2019-2024 consta de tres ejes generales que permiten agrupar los problemas públicos identificados a través del Sistema Nacional de Planeación Democrática y tres transversales que corresponden a temas comunes a los problemas públicos identificados, en los primeros se encuentran: 1. Justicia y Estado de Derecho, para promover la construcción de paz, el acercamiento del gobierno a la gente y el fortalecimiento de las instituciones del Estado Mexicano; 2. Bienestar, que busca asegurar que toda la población tenga acceso a una vivienda digna, promoviendo el pleno ejercicio de los derechos sociales, además, va enfocado en garantizar protección social para personas que viven en situaciones de vulnerabilidad; 3. Desarrollo Económico, cuya finalidad es garantizar el uso eficiente y responsable de recursos y la generación de los bienes, servicios y capacidades humanas para crear una economía fuerte y próspera (Centro Nacional de Control de Energía, 2019). 


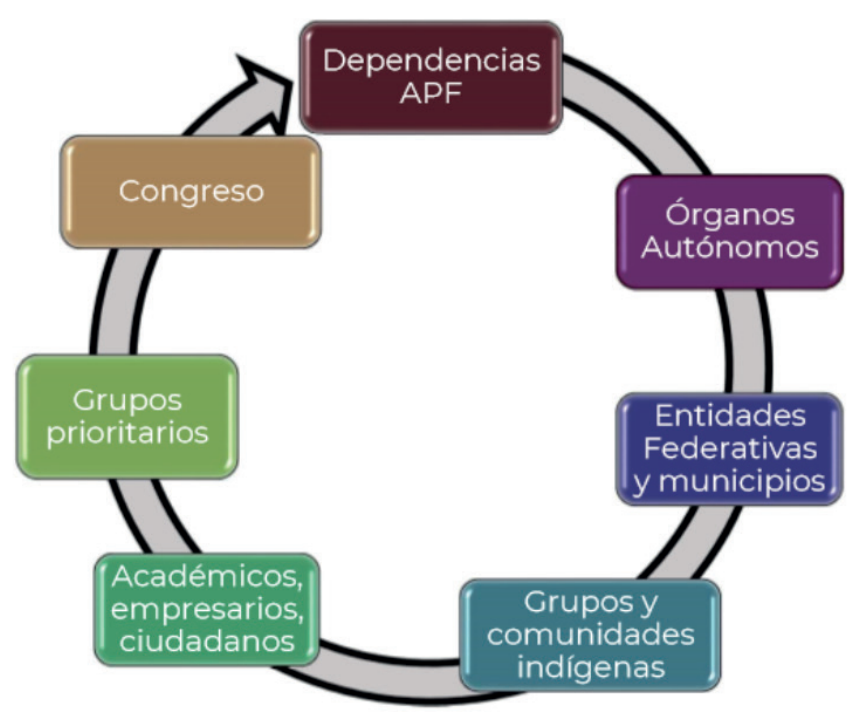

Fuente: Imagen tomada de https://opezdoriga.com/wp-content/uploads/2019/02/pnd-2019-2024-anteproyecto.pdf.

Para cada eje general se plantea un objetivo que refleja el fin último de las políticas propuestas en cada una de ellas; además, cada eje general se integra por el número de objetivos que corresponden a los resultados esperados, factibles y medibles que serán implementados en las políticas públicas propuestas.

Asimismo, en los ejes generales también se han fijado transversales: 1. Igualdad de género, no discriminación e inclusión, que busca incluir en las políticas públicas las perspectivas de género, intercultural, generacional y de desarrollo territorial; 2. Combate a la corrupción y mejora de la gestión pública, cuyo objeto es que las políticas públicas estén encaminadas a eliminar la corrupción y garantizar la eficiencia de la administración pública; y 3 . Territorio y desarrollo sostenible, para reconocer las consecuencias a futuro y lograr que las políticas públicas tengan un enfoque de desarrollo basado en la viabilidad económica, financiera, social y ambiental (Centro Nacional de Control de Energía, 2019).

Estos ejes transversales corresponden a tres problemas relevantes en el país que son comunes entre todos los temas de política pública que se busca atender, derivado de lo anterior, en términos del artículo $22^{\circ}$ de la Ley de Planeación, se pretende que los programas procedentes de este plan, así como los programas presupuestarios atiendan estos ejes con base en lineamientos de política pública diseñados específicamente para cada uno.

La importancia de estudiar el PND radica en que dicho documento al identificar los problemas principales que afectan a la sociedad en general, marca el rumbo de las políticas públicas que serán diseñadas con la intención de implementar acciones tendientes a resolver esos problemas plenamente identificados y así transformar la vida pública del país garantizando el bienestar a la población, lograr reducir las brechas de pobreza y desigualdad restableciendo un Estado de derecho con justicia que impulse el desarrollo económico sostenible. 
Imagen 2. Integración del PND

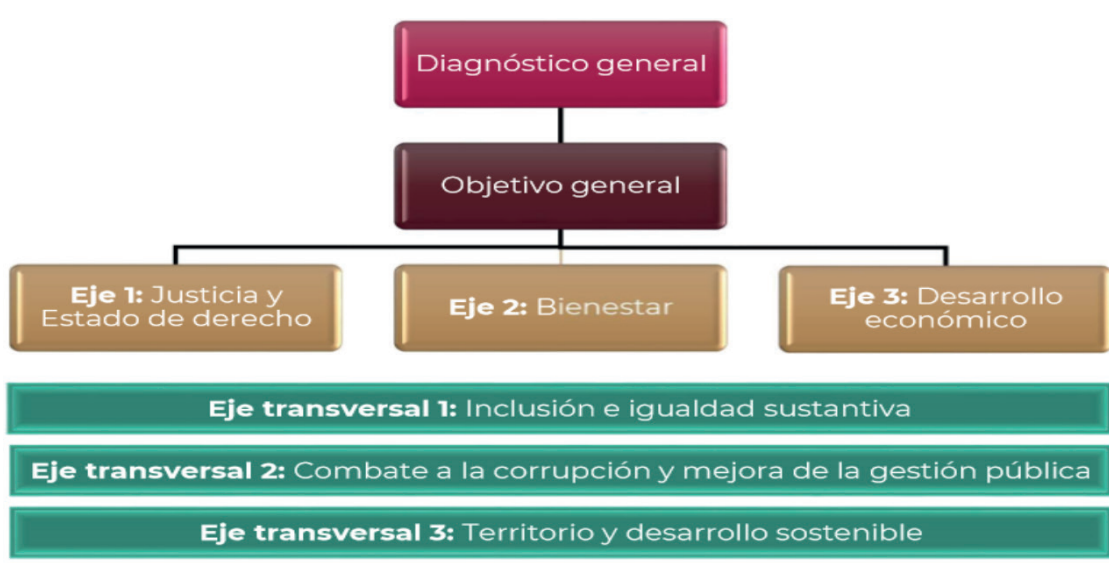

Fuente: Imagen tomada de https://opezdoriga.com/wp-content/uploads/2019/02/pnd-2019-2024-anteproyecto.pdf.

Derivado de lo anterior, resulta trascendente comprender el contexto en el que surgen las políticas públicas, una aproximación sobre su concepto, principales características y demás información que permita una mayor comprensión de la estrecha vinculación existente entre el PND y las políticas públicas.

\section{EL ESTUDIO DE LAS POLÍTICAS PÚBLICAS}

El estudio de las políticas se inició en 1950, en Estados Unidos, cuando algunos científicos sociales comenzaron a interesarse cada vez más por la toma de decisiones en y para el ámbito público. El trabajo de Harold Lasswell (2007), en particular, es el que con el tiempo se reconocería como la semilla originaria de lo que hoy se conoce como el enfoque de políticas públicas.

La aportación de Lasswell puede resumirse en dos aspectos principales que vinculan a las políticas públicas con la democracia en el siguiente tenor:

a) Comprender que el dar respuesta a las interrogantes planteadas por las ciencias de políticas implica aclarar tanto las metas que se persiguen con la acción gubernamental, como los valores que la sustentan; y

b) Visualizar a las políticas públicas como el puente entre los tomadores de decisión en el gobierno, los académicos de múltiples disciplinas y prácticamente cualquier otro actor que pueda aportar conocimiento valioso para la resolución de problemas específicos y trascendentes, esto es, problemas públicos, en el más amplio sentido del término (Arellano y Blanco, 2016, p. 31). 
Actualmente no contamos con una definición de políticas públicas única y universal, no obstante, diversos autores han retomado el espíritu democrático estudiado por Laswell y se han aventurado a exponer lo que se debe entender por este concepto.

Tal es el caso de Luis Aguilar, quien aborda este tema y entiende a las políticas públicas como "decisiones de gobierno que incorporan la opinión, la participación, la corresponsabilidad y el dinero de los privados, en su calidad de ciudadanos, electores y contribuyentes" (Arellano y Blanco, 2016, p. 36).

Las políticas públicas son decisiones y acciones legítimas de gobierno que se generan a través de un proceso abierto y sistemático de deliberación entre grupos, ciudadanos y autoridades con el fin de resolver, mediante instrumentos específicos, las situaciones definidas y construidas como problemas públicos (Arellano y Blanco, 2016, p. 31).

De las definiciones anteriores, se rescatan algunos aspectos centrales y se entiende a la política pública como toda acción gubernamental intencional, cuya meta es realizar un objetivo de interés para solucionar problemas públicos y atender demandas sociales, en este tenor, se debe tomar en cuenta que la eficiencia de las políticas públicas depende de las características o requisitos con que son diseñadas.

Las características mínimas que se consideran para hablar de una verdadera política pública son por lo menos cuatro, a saber:

1. Son llevadas a cabo por autoridades públicas legítimamente constituidas.

2. Surgen como respuesta a una situación entendida como un problema que afecta el interés público, por lo que debe resolverse empleando recursos gubernamentales, en mayor o menor medida.

3. Se materializan mediante mecanismos concretos, privilegiando el interés de la comunidad por encima de los intereses particulares.

4. Todo lo anterior, además, tiene como supuesto fundamental la discusión y el acuerdo entre el gobierno y la ciudadanía, respecto de qué debe resolverse, mediante qué mecanismos concretos y con qué efectos esperados. Así, al escoger una alternativa se están abandonando otras, al elegir un método de solución se están dejando de lado otros, por lo que estos procesos de "no-decisión" son también importantes (Arellano y Blanco, 2016, pp. 36-37).

Ahora bien, después de analizar este listado de características, resulta fácil percatarse que la realidad de las políticas públicas en nuestro país dista en gran medida de la forma en que debieran pensarse y formularse, por lo que se debe rescatar la necesidad de comprender las causas, razones, consecuencias de las acciones del gobierno, sobre todo de reconocer la imperiosa necesidad de que en estos procesos se vea reflejada la multiplicidad de ideas y puntos de vista, lo cual sólo puede darse en el marco de una democracia, tanto deliberativa como participativa.

En el caso del Estado Mexicano, durante varias décadas las decisiones de gobierno en su mayoría han sido tomadas de manera unilateral y autoritaria en los diferentes niveles de gobierno, olvidando que el entorno social democrático es el único en el que las políticas públicas pueden desarrollarse, ahora bien, con la implementación efectiva del PND como instrumento de participación ciudadana se 
pretende robustecer la democracia como simiente en el que pudiera prosperar alguna política pública que solucione, tienda al éxito, no al fracaso, como lo han hecho en escenarios anteriores, para ello, es necesario identificar y comprender el proceso de creación de una política pública, ya que ello permite identificar los momentos en que se pueden cometer errores y sobre todo la forma en cómo evitarlos.

\section{CREACIÓN DE POLÍTICAS PÚBLICAS}

De forma general, se mencionan cinco etapas que componen la creación (ideal) de toda política pública, a saber:

1. Entrada en la agenda gubernamental: el posicionar una situación en la agenda pública, y luego en la gubernamental, es una condición necesaria para la existencia de cualquier política pública, en la medida en que implica que ésta se reconoce como un problema; y no cualquiera, sino uno que amerita la atención del gobierno. La agenda pública es "el conjunto de asuntos elevados al estatus de problema público, [mientras que] la agenda gubernamental [es el] subconjunto del primero efectivamente susceptible de atención por parte del Estado a través de políticas públicas" (Casar y Maldonado, 2008, pp. 211-212).

2. Definición del problema: se entienden los procesos mediante los cuales un problema, ya aceptado como público y colocado en la agenda, es estudiado en términos de sus probables causas, componentes y consecuencias (Aguilar, 2007, p. 52). Es decir, se trata de comprender la naturaleza del problema con el fin de proponer soluciones acordes, bien informadas y con la mayor probabilidad de impactar positivamente sobre la situación considerada como negativa, cuando ello es factible.

3. Diseño: después de que se ha definido una situación social como problema público, y tras haberse estudiado éste en términos de sus elementos causales, se llega a la etapa del diseño de la política. Ésta no es otra cosa que la formulación de la estrategia que se seguirá para intentar resolver el problema en su contexto particular, entendiendo por estrategia el conjunto de instrumentos coherentemente integrados, así como las restricciones que estos enfrentarían. En suma, se trata de establecer una relación entre los medios manipulables y los objetivos alcanzables dadas las condiciones del entorno (Aguilar, 2007, p. 235).

4. Implementación: es el proceso de reunir y poner en su lugar todos los elementos necesarios para que una política funcione y se enfrente a una realidad más compleja de lo que cualquier diseño puede modelar, en la que lo único seguro es la propia incertidumbre.

5. Evaluación: es el momento último de toda política pública en donde se determinan las fortalezas y debilidades de los programas y proyectos públicos, una vez que estos se llevaron a cabo. Se trata de un acto inherentemente normativo, esto es, una cuestión de apreciación o juicio político (Hart, 2005, pp. 319-335).

El abordaje de estas etapas permite analizar las políticas públicas detalladamen- 
te para comprender mejor su construcción, desarrollo y alcances, estudiarlas así es sólo una propuesta metodológica, algunos autores identifican tres fases: una de diseño, una de implementación y otra de evaluación, no obstante, el objetivo es aportar elementos que permitan contrastar este proceso con la realidad en la que se desarrollan las políticas públicas, por lo menos en nuestro país e identificar la fase a la cual se debe el éxito o fracaso.

\section{Imagen 3. El proceso o ciclo de políticas públicas}

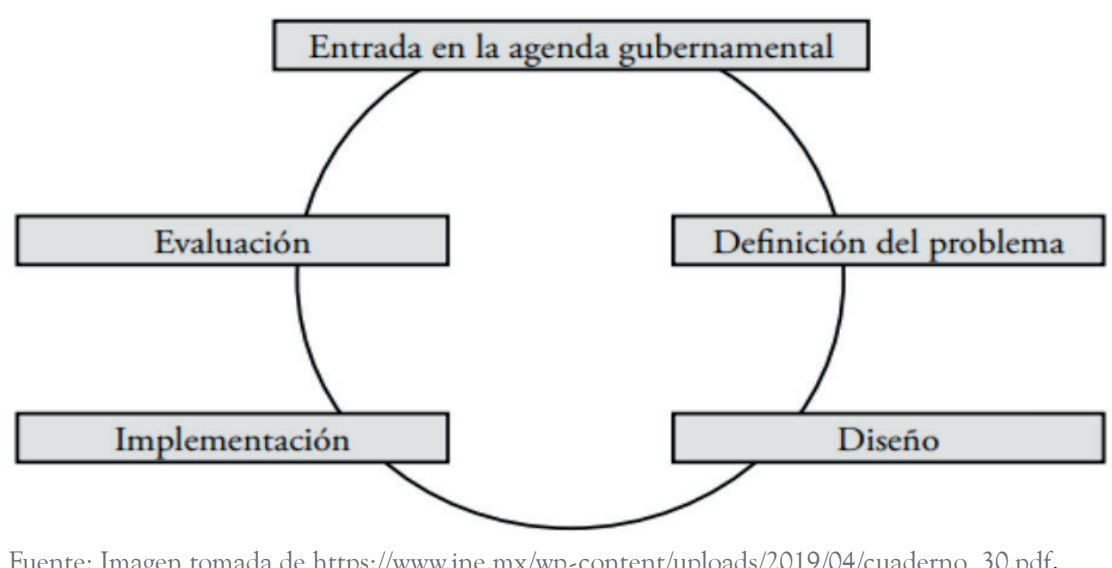

\section{LA REALIDAD DE LAS POLÍTICAS PÚBLICAS EN MÉXICO}

En nuestro país, las políticas públicas se han enfocado en dar soluciones genéricas a la mayoría de los problemas públicos: más presupuesto, programas y estructura administrativa, todo ello en el marco de un gran plan nacional, de ahí que los resultados globales indican que en general las decisiones tomadas hayan sido malas y costosas al no contar con propuestas de distintos agentes que propongan alternativas y alerten sobre los posibles efectos no deseados verbigracia crisis económicas y políticas, para ello la democracia participativa activa resulta fundamental.

Se debe tomar en consideración también que durante años los distintos gobiernos han planteado distintas estrategias de desarrollo social y productivo atendiendo a las preocupaciones y problemáticas comunes, no obstante, el mal diseño de sus políticas públicas no ha permitido abatir y erradicar los problemas que hasta hoy día nos aquejan, ello no es más que el reflejo de políticas públicas fallidas.

Empero, también debemos reconocer que existe un caso de éxito en el caso del programa Prospera, que es el único vigente de transferencia condicionada de México reconocido por la Comisión Económica para América Latina y el Caribe (CEPAL, 2019), y que vale la pena estudiar, toda vez que en él se concentran las características esenciales de una política pública ideal, lo que demuestra que en nuestro país sí es posible la implementación de más políticas públicas que tengan 
como ejes rectores el caso de este programa, cuyo objetivo es articular y coordinar la oferta institucional de programas y acciones de política social, fomento productivo, generación de ingresos, bienestar económico, inclusión financiera y laboral, educación, alimentación y salud.

Este programa está dirigido a la población (familias) en situación de pobreza extrema, a quienes bajo esquemas de apoyos se les permite mejorar condiciones de vida al asegurarles el goce de sus derechos sociales generando igualdad de oportunidades.

Este programa ofrece dos esquemas de apoyo, a saber:

1. El esquema de apoyos con corresponsabilidad, en el que las familias pueden recibir los apoyos de todos los componentes del programa debido a que la cobertura y capacidad de atención de los servicios de educación y salud permiten operar de forma simultánea los componentes educativos, de salud y alimentario; $y$

2. Esquema de apoyos sin corresponsabilidad, en el que la cobertura y capacidad de atención de los servicios de educación y salud no permiten operar de forma simultánea los componentes educativos, de salud y alimentario, por lo cual las familias sólo pueden recibir los apoyos de los componentes alimentario, de vinculación y de educación superior, sin estar sujetas a acciones de corresponsabilidad para recibir los apoyos del programa (Base de datos de programas de protección social no contributiva en América Latina y el Caribe, 2019).

El programa complementa sus acciones con otros programas sociales que ofrecen acceso a servicios básicos de vivienda y seguridad social, por ello es uno de los programas más estudiados y evaluados rigurosamente por instituciones externas, los estudios han demostrado impactos positivos del programa en tasas de escolarización y en el nivel de educación conseguido por los niños, mejoras significativas en el estado nutricional y mayor prevención de salud, los estudios muestran el importante papel del Programa en la reducción de la pobreza por ingresos en las zonas rurales, atribuyéndole hasta un tercio de ésta (Base de datos de programas de protección social no contributiva en América Latina y el Caribe, 2019).

Múltiples son las fortalezas de este programa y bien vale la pena enunciarlas, la población a la cual se dirige está muy bien definida, los mecanismos de priorización son claros y transparentes, el suministro de dinero en efectivo entregado de forma directa a las familias incentiva a las mismas a enviar a los menores a la escuela, acudir a las consultas al centro de salud, para no perder el estímulo que les permite cubrir gastos de alimentación y dota a las futuras generaciones de herramientas para romper con el círculo de pobreza.

Además, dado que su presencia territorial dentro del país es muy amplia, se permite mantener comunicación directa con zonas marginadas y remotas beneficiándolas también, es esta misma presencia lo que les permite identificar y resolver los principales problemas que se les presenten.

Un aspecto fundamental es que cuenta con una extraordinaria agenda de evaluación, con lo que se ha visto favorecido el diseño a lo largo del tiempo en que ha sido permanente su implementación.

Este caso de éxito hace identificables las características que debe contener una política pública deseable, demostrando que, con planeación, participación, 
transparencia es posible gobernar con eficacia, es en este tenor que resulta de interés analizar el PND 2019-2024, por ser el documento base que guiará el gobierno en torno a las políticas públicas que se implementan en nuestro país.

\section{MARCO JURÍDICO DEL PND}

El artículo 21 de la Declaración Universal de los Derechos Humanos consagró el derecho de toda persona a participar en el gobierno de su país, sea directamente o por medio de representantes elegidos libremente, así como a participar en las funciones públicas en condiciones de igualdad (Arellano y Blanco, 2016, p. 9).

En ese tenor es que la Constitución Política de los Estados Unidos Mexicanos (CPEUM) establece en el artículo 26 que "el Estado organizará un sistema de planeación democrática del desarrollo nacional que imprima solidez, dinamismo, competitividad, permanencia y equidad al crecimiento de la economía para la independencia y la democratización política, social y cultural de la nación”.

Asimismo, la CPEUM mandata que la planeación será democrática y deliberativa, mediante mecanismos de participación, con el objeto de recoger las aspiraciones y demandas de la sociedad para incorporarlas al PND, al que se sujetarán obligatoriamente los programas de la APF.

En este tenor se creó la Ley de Planeación, que establece plazos y procedimientos para la formulación y publicación del PND, cabe mencionar que dicha ley fue reformada el 2 de febrero de 2018 para adicionar un elemento sustantivo al procedimiento de publicación, ahora de conformidad con el artículo 21 de la ley, el ejecutivo debe enviar a la Cámara de Diputados el PND para su aprobación (el Plan se presenta en dos documentos: uno que incluye los principios rectores de política y otro donde se describe los objetivos generales, metas y sus estrategias), órgano que debe resolver dentro de los próximos dos meses a la fecha de su presentación, en este sentido, la tarea consiste en verificar que dicho instrumento incluya los fines del proyecto nacional contenidos en la CPEUM.

Además, el artículo $20^{\circ}$ de la ley en comento señala que en el ámbito del Sistema Nacional de Planeación Democrática tendrá lugar la participación y consulta de los diversos grupos sociales, con el propósito de que la población exprese sus opiniones para la elaboración, actualización y ejecución del pND y los programas que de este deriven, lo que dio sustento al Sistema Nacional de Planeación Democrática y la consulta ciudadana (Proceso de consulta que el Gobierno de México llevó a cabo a los diversos grupos sociales del país, se originaron opiniones que enriquecieron los diagnósticos y el plan de acción que se implementará durante los próximos seis años).

\section{ETAPAS DEL PND}

La elaboración del PND se lleva a cabo a través de tres etapas, a saber:

ETAPA 1

- Se realiza un diagnóstico de la situación actual y se hace una perspectiva a largo plazo de los temas prioritarios con base en información cualitativa y cuantitativa, para ello se utilizan indicadores clave del Sistema Nacional de Información 
Estadística y Geográfica (SNIEG) o Análisis de la acción gubernamental que se ha implementado, brechas y rezagos que persisten y áreas de oportunidad para atenderlos.

- Se hace una identificación de problemas públicos prioritarios, de sus causas y efectos.

\section{ETAPA 2}

- Se identifican los objetivos, que deben ser alcanzados para resolver o mitigar un problema público.

- Se plantean las estrategias y medios requeridos para solucionar las causas que generan el problema público.

- Se analizan los indicadores y metas a través de métricas cuantitativas para evaluar y dar seguimiento a los objetivos.

\section{ETAPA 3}

- Se llevan a cabo las consultas con actores relevantes para enriquecer el diagnóstico y los objetivos prioritarios a través de foros nacionales, estatales y especiales (con grupos indígenas y personas con discapacidad), con la finalidad de elevar las preocupaciones y problemáticas de grupos, cuyas voces han sido poco escuchadas, además también se llevan a cabo mesas sectoriales.

El proceso de consulta en esta ocasión tuvo como instrumento una encuesta ciudadana por internet y se desarrolló del 28 de febrero al 15 de abril de 2019 en la forma siguiente:

- Se llevaron a cabo 3 foros nacionales que se denominaron "Planeando Juntos la Transformación de México" en los que se realizó una consulta en relación a los 3 ejes, estos foros fueron presididos por el presidente y contaron con la participación de distintos grupos de la población (indígenas, mujeres y personas con discapacidad).

- Se realizaron 32 foros estatales, uno por cada entidad federativa en donde se discutieron los 3 ejes generales enfatizando el rol de los ejes transversales para atender los objetivos.

- Se llevaron a cabo 87 mesas sectoriales, cuyo carácter es opcional para enriquecer y retroalimentar el diagnóstico y las estrategias de las Secretarías, y ahondar en algún tema prioritario para las dependencias.

- Se llevaron a cabo 29 foros especiales en México y 13 en Estados Unidos de América para incorporar a la planeación nacional la visión de grupos prioritarios (grupos y comunidades indígenas y personas con discapacidad).

- Además, se lanzó una consulta digital, una encuesta realizada a niñas, niños, adolescentes, hubo recepción de propuestas de política pública a través de correo electrónico oficial, de forma tanto virtual como física (SHCP, 2019). 


\section{ETAPA 4}

Se redacta el documento final integrando, enriqueciendo y modificando las ideas de las propuestas preliminares del Gobierno con los insumos de los foros, las propuesta y consultas en línea derivadas de la etapa que antecede.

\section{ETAPA 5}

Una vez que el Ejecutivo Federal cuenta con el documento final, debe presentarlo y someterlo a la Cámara de Diputados para su discusión, aprobación y en su caso publicación.

Cabe mencionar que como se expuso, derivado de la reforma del año anterior, ésta es la primera ocasión en que se realiza esta etapa, en el caso particular del PND 2019. 2024, tuvo como la fecha límite para su presentación el 30 de abril del año en curso, fue aprobado y publicado en el Diario Oficial de la Federación el pasado doce de julio, encontrándose disponible para su consulta en la siguiente dirección electrónica: http://www.dof.mx/nota_detalle.php?codigo $=5565599 \&$ fecha $=12 / 07 / 2019$.

\section{PRINCIPIOS RECTORES DEL PND}

El PND 2019-2024 se sustenta en doce puntos centrales, recogidos del consenso nacional y que tienen como centro la convicción de que el quehacer en su conjunto (el económico, político, social y cultural) debe ser orientado a alcanzar el bienestar de la población, para lo cual se plantea que el gobierno recupere su función de árbitro protegiendo los derechos individuales y colectivos en su papel de impulsor y conductor de la economía.

Para el cumplimiento de su objetivo, este plan rige su política de desarrollo por los siguientes doce principios:

1. Honradez y honestidad: para acabar con la corrupción en toda la administración pública.

2. No al gobierno rico con pueblo pobre: para poner fin a los dispendios con una política de austeridad republicana.

3. Nada al margen de la ley: por encima de la ley, nadie: Lo que se traduce en respetar lo previsto por la CPEUM, observando los derechos sociales, colectivos y sociales, empezando por los derechos humanos, poniendo fin a la represión política y poniendo fin a los privilegios ante la ley y cese de los fueros.

4. Economía para el bienestar: el objetivo de la política económica es generar bienestar para la población, lo que implica un crecimiento con austeridad y sin corrupción, disciplina fiscal, cese del endeudamiento, respeto a las decisiones autónomas del Banco de México, creación de empleos, fortalecimiento del mercado interno, impulso al agro, a la investigación, la ciencia y la educación.

5. El mercado (Ríos Ruíz, 2010), no sustituye al Estado: propone que el Estado recuperare su fortaleza como garante de la soberanía, la estabilidad, el estado de derecho, árbitro de los conflictos, generador de políticas públicas coherentes, articulador de los propósitos nacionales. 
6. Por el bien de todos, primero los pobres: recuperación del principio de empatía social con los miembros más débiles y desvalidos que es factor indispensable de cohesión.

7. No dejar a nadie atrás, no dejar a nadie fuera: implementación de un modelo de desarrollo respetuoso de los habitantes, del hábitat, equitativo, orientado a subsanar, no a agudizar las desigualdades, defensor de la diversidad cultural y del ambiente natural, sensible a las modalidades, singularidades económicas regionales y locales, consciente de las necesidades de los habitantes futuros del país.

8. No hay paz sin justicia: implementación de un nuevo paradigma en materia de paz, seguridad que tenga como prioridad restarle base social a la criminalidad mediante la incorporación masiva de jóvenes al estudio, al trabajo para apartarlos de conductas antisociales; recuperación del principio de reinserción social; fin de la "guerra contra las drogas", adopción de una estrategia de prevención, tratamiento de adicciones; impulso a procesos regionales de pacificación con esclarecimiento, justicia, reparación, garantía de no repetición, reconciliación nacional, medidas contra el lavado de dinero e inteligencia policial, para ello la Guardia Nacional funge como policía de paz y proximidad, con presencia permanente en todo el territorio.

9. El respeto al derecho ajeno es la paz: respeto de los principios de no intervención, autodeterminación, relaciones con todos los pueblos basadas en la cooperación para el desarrollo, solución pacífica de los conflictos mediante el diálogo y rechazo a la violencia y a la guerra, respeto a los derechos humanos.

10. No más migración por hambre y violencia (Ríos Ruíz, 2019): por una política encaminada a ofrecer a todos los ciudadanos las condiciones adecuadas para que puedan vivir con dignidad y seguridad en su país de origen. A los extranjeros que llegan a territorio mexicano se les brindará respeto a sus derechos, hospitalidad y la posibilidad de que construyan aquí una nueva vida.

11. Democracia (Ríos Ruíz, 2019), significa el poder del pueblo: implementación de una democracia participativa para socializar el poder político e involucrar a la sociedad en las grandes decisiones nacionales.

12. Ética, libertad y confianza: Los principios éticos y civilizatorios serán las claves del nuevo pacto social y del modelo de desarrollo para México (Presidencia de la República, Plan Nacional de Desarrollo, 2019).

Estos doce puntos rectores permean en todo el PND y se ven reflejados en los ejes generales y transversales.

\section{PROGRAMAS DERIVADOS DEL PND}

Los programas de gobierno, que en el caso del PND 2019-2024 son 44, fungen como uno de los instrumentos que ponen en marcha las acciones trazadas por las políticas públicas con el fin de solucionar problemas públicos específicos y prioritarios que se identifican con el estudio realizado para la creación del PDN, en este tenor podemos comprender que del PDN se derivan las políticas públicas y de éstas los programas de gobierno.

1. PND (Identificación de problemas) $\rightarrow$ 2. Políticas Públicas (Soluciones a los problemas planteados) $\rightarrow$ 3. Programas de Gobierno (Acciones específicas para solucionar cada problema). 
Estos programas tienen sustento en el artículo 22 de la Ley de Planeación (Ley de Planeación, 2018), cabe puntualizar que estos programas serán elaborados sin perjuicio de aquellos que prevean las leyes o determine el presidente de la República con posterioridad.

A continuación se enlistan (Gaceta Parlamentaria, 2019) los programas, no sin antes hacer mención que de acuerdo con lo previsto por los artículos 16 y 17 de la Ley de Planeación, las dependencias de la APF deberán elaborar sus programas sectoriales y las entidades paraestatales sus correspondientes programas institucionales.

Programa Regional de Desarrollo y Bienestar de la Cuenca Balsas-Pacífico Sur

Programa Nacional de Derechos Humanos

Programa Nacional de Población

Programa Especial de Política Migratoria

Programa Nacional de Cultura de Paz y Prevención Social del Delito para la Reconstrucción del Tejido Social

Programa Especial para Vivir la Democracia: Impulso del Desarrollo Democrático y la Participación Social

Programa Nacional para la Igualdad y No Discriminación

Programa Nacional de Búsqueda y Localización

Programa Integral para Prevenir, Atender, Sancionar y Erradicar la Violencia contra las Mujeres

Programa Nacional de Protección de Niñas, Niños y Adolescentes

Programa Nacional para Prevenir, Sancionar y Erradicar los Delitos en Materia de Trata de Personas y para la Protección y Asistencia a las Víctimas de estos Delitos

Programa de Atención Integral a Víctimas

Programa Nacional de Seguridad Pública

Programa para la Seguridad Nacional

Programa Nacional de Prevención Social de la Violencia y la Delincuencia

Programa Nacional de Protección Civil

Programa de Cooperación Internacional para el Desarrollo

Programa Regional para el Desarrollo del Istmo de Tehuantepec

Programa Nacional para Profundizar la Inclusión Financiera

Programa Especial Concurrente para el Desarrollo Rural Sustentable

Programa de Manejo Sustentable del Agua y el Suelo Agrícola en las regiones del Noroeste y Noreste de México

Programa Especial para la Productividad y la Competitividad 
Programa Nacional de Cultura Física y Deporte

Programa para la Transformación de la Educación Superior

Programa Especial para Formular y Encausar una Política General sobre el Futuro del Trabajo

Programa Nacional de Vivienda

Programa Nacional de Ordenamiento Territorial y Desarrollo Urbano

Programa Nacional de Suelo

Programa Especial de Cambio Climático

Programa Nacional Hídrico

Programa Nacional Forestal

Programa Nacional para la Prevención y Gestión Integral de los Residuos

Programa Nacional para la Prevención y Gestión Integral de los Residuos de Manejo Especial

Programa Nacional de Remediación de Sitios Contaminados

Programa Nacional de Desarrollo Social

Programa Nacional para el Desarrollo y la Inclusión de las Personas con Discapacidad

Programa Nacional de Juventud

Programa de Fomento a la Economía Social

Programa Nacional de Mejora de la Gestión Pública y Combate a la Corrupción y la Impunidad

Programa Especial de Ciencia, Tecnología e Innovación

Programa para el Desarrollo de la Bioseguridad y la Biotecnología

Programa de Atención Integral a Víctimas

Programa Nacional para la Igualdad entre Hombres y Mujeres

Trece Programas Hídricos Regionales

Resulta preciso mencionar en relación al listado anterior de los programas que derivaron del PND que los mismos aún no cuentan con una agenda ni líneas de acción para su puesta en marcha e implementación que se haya dado a conocer de forma pública, lo que resulta comprensible dada la reciente publicación del PND en el DOF como se refirió en líneas anteriores.

Aun así se destaca la próxima implementación del Programa Nacional de Seguridad Pública dada la importancia que tiene la seguridad de la población para alcanzar el bienestar social integral, con ello se reconoce que el alcanzar esto es la razón primordial de la existencia del poder público y que las instituciones hasta ahora encargadas de ello han sido incapaces de utilizar sus potestades, pues han fallado en su responsabilidad de preservar la vida, la integridad y la propiedad de 
las personas y las poblaciones, este programa reconoce la crisis existente y que se ha degradado la calidad de vida. Esto es un claro ejemplo de política pública a la que valdría la pena darle seguimiento.

\section{EL PND Y LOS OBJETIVOS DE DESARROLLO SOSTENIBLE}

En 2015, la Organización de Naciones Unidas (ONU) aprobó la Agenda 2030 sobre el desarrollo sostenible estableciendo 17 Objetivos de Desarrollo Sostenible (ODS, 2019) que han sido adoptados por más de 150 países y es en este tenor que los objetivos del eje general del PND 2019-2024 se encuentran en armonía con estos principios y se vinculan en un $40.0 \%$, ya que el número de objetivos relacionados entre ambas partes asciende a 204.

Los 17 ODS que a continuación se enuncian abarcan diversos temas como justicia, instituciones sólidas, pobreza, derechos sociales básicos, crecimiento económico, innovación, inclusión social, desigualdad y sostenibilidad ambiental.

1. Poner fin a la pobreza en todas sus formas en todo el mundo.

2. Poner fin al hambre, lograr la seguridad alimentaria y la mejora de la nutrición y promover la agricultura sostenible.

3. Garantizar una vida sana y promover el bienestar para todos en todas las edades.

4. Garantizar una educación inclusiva, equitativa y de calidad y promover oportunidades de aprendizaje durante toda la vida para todos.

5. Lograr la igualdad entre los géneros y empoderar a todas las mujeres y las niñas.

6. Garantizar la disponibilidad de agua y su gestión sostenible y el saneamiento para todos.

7. Garantizar el acceso a una energía asequible, segura, sostenible y moderna para todos.

8. Promover el crecimiento económico sostenido, inclusivo y sostenible, el empleo pleno y productivo y el trabajo decente para todos.

9. Industria, innovación e infraestructuras.

10. Reducir la desigualdad en y entre los países.

11. Lograr que las ciudades y los asentamientos humanos sean inclusivos, seguros, resilientes y sostenibles.

12. Garantizar modalidades de consumo y producción sostenibles.

13. Adoptar medidas urgentes para combatir el cambio climático y sus efectos. 
14. Conservar y utilizar en forma sostenible los océanos, los mares y los recursos marinos para el desarrollo sostenible.

15. Gestionar sosteniblemente los bosques, luchar contra la desertificación, detener e invertir la degradación de las tierras y detener la pérdida de biodiversidad.

16. Promover sociedades justas, pacíficas e inclusivas.

17. Revitalizar la alianza mundial para el desarrollo sostenible (Ley de Planeación, 2018).

A continuación, a través de la siguiente matriz (Ley de Planeación, 2018) se desglosa de forma particular las relaciones de los objetivos de cada eje central con los ODS.

Imagen 4. Matriz comparativa de los ejes generales que integran el PND y su relación con los ODS

Vinculación entre los Objetivos del PND y los Objetivos de Desarrollo Sostenible

\begin{tabular}{|c|c|}
\hline Eje General & $\begin{array}{c}\text { Número de ODS relacionados } \\
\text { con los objetivos del Eje General } \\
\text { del PND }\end{array}$ \\
\hline Justicia y Estado de derecho (9 objetivos) & 40 \\
\hline Bienestar (11 objetivos) & 80 \\
\hline Desarrollo económico (10 objetivos) & 84 \\
\hline Total (30 objetivos) & 204 \\
\hline
\end{tabular}

Fuente: Imagen tomada de http://gaceta.diputados.gob.mx/PDF/64/2019/abr/20190430-XVIII-1.pdf.

Con los datos anteriores queda claro que poca relación guardan los objetivos del PND 2019-2024 con los ODS, que si bien no son jurídicamente obligatorios, se espera que los gobiernos los adopten como propios y establezcan marcos nacionales para el logro de los mismos (ODS, 2019), hecho que en este caso ocurre de forma precaria y que indiscutiblemente traerá consigo un rezago en cuanto a las actividades regionales de seguimiento y examen globales respecto a la atención que como país se da a estos objetivos.

Este análisis nos sirve como parámetro para reflejar la vinculación de nuestras políticas públicas derivadas del PND y la forma en que éstas comparten o no la visión y objetivos de la comunidad internacional respecto a las personas y planeta.

\section{CONCLUSIONES}

1. Las políticas públicas en nuestro país requieren un cambio de fondo, mismo que se debe gestar desde la planeación de las mismas a través del PND; los elementos para su creación requieren entre otras cosas, de diagnósticos eficientes sobre problemáticas sociales, intervención de la sociedad civil en las etapas de creación, presupuestación, implementación y por supuesto evaluación, implementando para cada etapa procesos de inclusión y deliberación con el objeto de que éstas sean integrales y cumplan su objetivo. 
2. El PND 2019-2024 como documento que debe recoger las estrategias para planificar, conducir y orientar las acciones públicas encaminadas a lograr el desarrollo social nacional, se limita a enunciar una serie de ambiciosos objetivos, pero no establece cómo es que habrán de materializarse y cómo se evaluará el desempeño de los mismos; habla de crecimiento, austeridad, lucha contra la corrupción, disciplina fiscal, cese de endeudamiento, creación de empleos y una serie de acciones más, sin que se diga cómo es que cada uno de esos anhelos serán hechos realidad, es decir, no se señalan acciones concretas, el desarrollo del documento carece de una valoración objetiva de las circunstancias en que se diagnostica la situación nacional, lo que da un sentido de subjetividad en el tratamiento de los temas.

3. De la lectura y análisis del PND se puede destacar que su contenido no está acorde con los elementos mínimos de un PND, tal como lo prevé la Ley de Planeación ni conforme a las definiciones contenidas en la Guía Técnica y Metodológica para la elaboración del PND 2019-2024 emitida por la Secretaría de Hacienda y Crédito Público (SHCP). En particular, como ya se apuntó no contiene objetivos, estrategias, indicadores, previsiones de gasto, estudios de factibilidad cultural ni tiempos de ejecución, lo que en su conjunto dificulta y en su caso imposibilita los procesos de evaluación sobre el grado de cumplimiento de metas y objetivos, que permitan conocer el impacto social tanto del Plan, como de los programas que de él derivan, es decir, las políticas públicas implementadas.

4. El PND desde ninguna perspectiva debe ser un documento de naturaleza teórica, su objeto es recoger los principios generales que definirán las acciones que cambiarán la realidad social, por lo que no debe hacerse de las prioridades nacionales un discurso, pues no se debe perder de vista que la forma en que se distribuyen los recursos públicos de la nación a través del presupuesto de egresos de la federación depende de este documento, ahí radica la importancia de dejar fuera del mismo los fanatismos personales y políticos.

\section{REFERENCIAS}

1. Arellano Gault, D., Blanco, F. (2016). Políticas públicas y democracia. Cuadernos de Divulgación de la Cultura Democrática. México: Instituto Federal Electoral.

2. Base de datos de programas de protección social no contributiva en América Latina y el Caribe (2018). Prospera. Programa de Inclusión Social (2014-2019). Recuperado de https://dds.cepal.org/bpsnc/programa?id=92.

3. Bovens, M., Hart, P., Kuipers, S. (2005). The Politics of Policy Evaluation. En Moran, M. Rein, M. y Goodin, R.E. (eds.). The Oxford Handbook of Public Policy. Oxford: Oxford University Press.

4. Casar, M. A., Maldonado, C. (2018). Formación de agenda y proceso de toma de decisiones. Una aproximación desde la ciencia política. En Mau- 
ricio y Cejudo Guillermo (comps.). Problemas, decisiones y soluciones. Enfoques de política pública. México: Fondo de Cultura Económica, CIDE.

5. Centro de Estudios de Finanza Públicas (2019). Aspectos relevantes del Plan Nacional de Desarrollo. Palacio Legislativo de San Lázaro, Ciudad de México. Recuperado de http://www.cefp.gob.mx/publicaciones/documento/2019/cefp0112019.pdf.

6. Centro Nacional de Control de Energía (2019). Plan Nacional de Desarrollo 2019-2024. Gobierno de México. Recuperado de https://www. gob.mx/cenace/acciones-y-programas/plan-nacional-de-desarro1lo-2019-2024-195029.

7. Constitución Política de lo Estados Unidos Mexicanos (CPEUM) (2019). Recuperado de https://mexico.justia.com/federales/constitucion-politica-de-los-estados-unidos-mexicanos/titulo-primero/capitulo-i/.

8. Gaceta Parlamentaria (2019). Recuperado de http://gaceta.diputados. gob.mx/PDF/64/2019/abr/20190430-XVIII-1.pdf.

9. Lasswell, H. (2007). La orientación hacia las políticas. En Luis F. Aguilar Villanueva (ed.), El estudio de las politicas públicas. México: Miguel Ángel Porrúa.

10. Ley de Planeación (2018). Recuperado de https://www.google.com/ur1 ? sa $=\mathrm{t} \&$ source $=$ web\&rct $=\mathrm{j} \& u r l=\mathrm{http}: / / \mathrm{www}$.diputados.gob.mx $/$ LeyesBiblio/pdf/59 160218.pdf\&ved=2ahUKEwiigc6e3671AhVMnKwKHbJFAIYQFjAA-egQIBRAB\&usg=AOvVaw0o411EuP6-ZK41O5skBW5A.

11. Objetivos de Desarrollo Sostenible (ODS) (2019). Impacto Académico. Recuperado de https://academicimpact.un.org/es/content/objetivos-de-desarrollo-sostenible.

12. Presidencia de la República (2019). Plan Nacional de Desarrollo. Recuperado de https://opezobrador.org.mx/wp-content/uploads/2019/05/ PLAN-NACIONAL-DE-DESARROLLO-2019-2024.pdf.

13. Ríos Ruiz, A. Á. (2010). Larectoría Económica Constitucional en la Política de la Globalización, Amicus Curiae. Recuperado de http://revistas. unam.mx/index.php/amicus/article/view/14585.

14. Ríos Ruiz, A. Á. (2019). Migración y comercio. Visión comparada México y Estados Unidos de América, Perfiles de las Ciencias Sociales. Recuperadode https://scholar.google.es/scholar?start $=20 \& q=\% 22 \mathrm{Alma}+\mathrm{de}+\mathrm{los}+\%$ C3\%81ngeles\%22++R\%C3\%ADos + Ru\%C3\%ADz\&hl=es\&as_sd$\mathrm{t}=0,5 \# \mathrm{~d}=$ gs_qabs\&u $=\% 23 \mathrm{p} \% 3 \mathrm{DdgXgdKDhXw0J}$.

15. Secretaría de Hacienda y Crédito Público (SHCP) (2019). Plan Nacional de Desarrollo 2019-2024. Recuperado de https://opezdoriga.com/ wp-content/uploads/2019/02/pnd-2019-2024-anteproyecto.pdf. 